\title{
A INCLUSÃO DE UMA NOVA FERRAMENTA PARA O ENSINO OPERACIONAL DE REDES DE DISTRIBUIÇÃO DE ENERGIA ELÉTRICA
}

\author{
Caio dos Santos ${ }^{1}$ - caiodos.santos@ hotmail.com \\ Marina Camponogara ${ }^{1}$ - camponogaramarina@gmail.com \\ Daniel Pinheiro Bernardon ${ }^{1}$ - dpbernardon@gmail.com \\ ${ }^{1}$ Centro de Excelência em Energia e Sistemas de Potência (CEESP) \\ Universidade Federal de Santa Maria (UFSM)
}

\begin{abstract}
RESUMO
Dentre as composições do sistema elétrico brasileiro, o segmento de distribuição é, sem dúvidas, o setor que recebe a maior quantidade de energia através das linhas de transmissão, e a distribui, de maneira pulverizada, aos consumidores. Devido à importância das redes de distribuição, sua operação é uma tarefa de grande responsabilidade, a qual exige todo o empenho do profissional responsável. Assim, sua formação ideal deve combinar o desenvolvimento de competências técnicas, habilidades, atitudes éticas, sociais e ambientais. No entanto, nem todos os segmentos da Engenharia Elétrica possuem instrumentos que permitam que a teoria seja implementada, em laboratório, de forma dinâmica, como por exemplo, os processos de planejamento e operação de sistemas elétricos de potência. Verifica-se que nesta área há uma carência de equipamentos de natureza experimental, onde o conhecimento prático é embasado apenas na reprodução de simulações, realizadas através de ferramentas computacionais. Assim, propõe-se neste trabalho uma análise de aplicabilidade de inserção de uma ferramenta experimental de ensino. Esta, deve ser capaz de reproduzir algumas das principais características operacionais de uma rede real, em laboratório, de forma a inovar e facilitar o ensino de processos de planejamento operacional de redes de distribuição de energia elétrica, fomentando os principais conceitos de métodos de aprendizagem baseados em problemas.
\end{abstract}

Palavras-chave: Métodos de otimização; redes de distribuição; ferramenta experimental.

\section{THE INCLUSION OF A NEW TOOL TO OPERATIONAL TEACHING OF ELETRICAL POWER DISTRIBUTION NETWORKS}

\begin{abstract}
Among the compositions of the Brazilian electrical system, the distribution segment is surely the sector that receives the most energy through the transmission lines, and distributes sprayed form to the consumers. Due its importance, the distribution network operation is a task of great responsibility, which requires all the commitment of the responsible professional. Thus, his ideal formation must combine the development of technical competence, skills and ethical, social and environmental attitudes. However, not all Electrical Engineering segments has instruments that allow to implement theories in laboratories for a dynamic form, such for example the processes of planning and operation of electric power systems. It seems that in this field there is a deficiency of experimental equipment where practical knowledge is grounded only in simulations reproduction, performed through computational tools. Thus, it is being proposed in this paper, an analysis of applicability if a teaching experimental tool to be inserted. This tool should be able to play some of the main operational characteristics of a real network, in laboratory, in order to innovate and facilitate the teaching of electricity distribution operational planning processes network, fostering the main concepts of learning methods based in problems.
\end{abstract}

Keywords: Optimization methods; distribution networks; experimental tool. 


\section{INTRODUÇÃO}

Dentre os segmentos do sistema elétrico de potência, o de distribuição de energia elétrica, atualmente, é responsável pela maior parcela de perdas de energia, devido as características de extensão de seus alimentadores (ALs) e a ramificação de sua rede secundária, ou de baixa tensão (Chang et al., 2003). Ainda, fatores relacionados a confiabilidade, agregam complexidade no processo de planejamento operacional pois, frequentemente, a rede é afetada pela interrupção no fornecimento de energia elétrica, exigindo a busca por soluções em um curto intervalo de tempo (Tsao et al., 2003 e Goel et al., 1999).

Dessa forma, aliado a necessidade de fornecer energia de qualidade e de se obter um sistema de alta confiabilidade, é comum o desenvolvimento, o aprimoramento e a aplicação de métodos preventivos e corretivos, que otimizem o processo operacional de uma rede de distribuição (RD). Estes, por sua vez, exigem capacidade intelectual e habilidade técnica dos profissionais responsáveis na busca de soluções, mesmo com o auxílio de todo aparato tecnológico utilizado. Por conta disso, ressalta-se a importância da manutenção de bons profissionais, preparando-os ainda no ambiente acadêmico.

Entretanto, o ensino relacionado à operação de RDs, atualmente, é fortemente embasado em simulações e reproduções de metodologias prontas, sendo consequência do conservadorismo de ensino e disciplinas totalmente conteudistas. Assim, o aluno acaba deixando de acompanhar o dinamismo envolvido neste processo, o que afeta diretamente o desenvolvimento de habilidades técnicas e não técnicas, como por exemplo, personalidade e agilidade processual.

Assim, inovar em métodos de ensino é fundamental para manutenção de bons profissionais, considerando as constantes mudanças ocorridas no mercado de trabalho. Diante dessa nova ideologia de ensino, trabalhos clássicos como o de Belhot (1997), já havia destacado a importância de esculpir nos futuros engenheiros a competência em identificar problemas, priorizá-los, analisar as alternativas e solucioná-los. Tais características são fundamentais, quando direcionadas ao contexto deste manuscrito.

Para tanto, cabe ao professor despertar o interesse do aluno pela pesquisa e a busca pelo novo, utilizando como principal ferramenta o conteúdo relacionado ao tema em que este é posto a lecionar. Quanto a academia, no cumprimento de seu papel, esta deve fornecer as ferramentas necessárias para que isso se torne possível, favorecendo o desenvolvimento de soluções técnicas e sociais (Macedo et al., 2012). Contudo, quando direcionados a sistemas elétricos, mais especificamente ao segmento de distribuição, nota-se que há carência de equipamentos didáticos, capazes de implementar em laboratório a teoria vista em sala de aula.

Sendo assim, visando sanar parte deste problema, o presente trabalho objetiva expor a experiência de implementação laboratorial de uma ferramenta educacional, desenvolvida para ser utilizada como meio de estudo para alunos de iniciação científica, no planejamento operacional de RDs. Com isso, busca-se fornecer conhecimento prévio de situações corriqueiras em que o aluno estará sujeito, a partir de um instrumento laboratorial, cuja proposta é aumentar o dinamismo de aulas direcionadas ao estudo de teorias clássicas relacionadas ao processo operacional de uma RD. 


\section{FORMULAÇÃO DO PROBLEMA}

Dentre as principais ferramentas utilizadas no processo operacional de uma RD, a reconfiguração é a mais importante, diante dos bons resultados obtidos, como apresentado nas pesquisas de Baran e Wu (1989), Cherkaoui (1993), Hsu (1992) e Civanlar (1988). Comumente, sua utilização se faz necessária por decorrência de dois fatores: $o$ restabelecimento de energia elétrica as unidades consumidoras, consequências de um sinistro; ou na manutenção da rede, visando a melhoria de desempenho do sistema (Bernardon et al., 2007).

Quando utilizada na manutenção, busca-se uma topologia de rede que apresente desempenho superior a atual (Pfitscher, 2013). Neste sentido, estudos são desenvolvidos com intuito de auxiliar na escolha da configuração ótima, bem como nas manobras necessárias para obtenção do objetivo, sendo este, na maioria dos casos, a redução de perdas de energia elétrica, como apresentado por Civanlar (1988).

Contudo, o operador nem sempre almeja um único objetivo, devendo, às vezes, otimizar diversos critérios operacionais. Para que isso se torne possível, há a necessidade do auxílio de métodos computacionais, que determinem as prioridades dentre os vários critérios, como abordado nas pesquisas desenvolvidas por Su (2003), Das (2006) e Hong (2005).

Atualmente, os procedimentos operacionais citados são previamente testados em ferramentas computacionais e, em seguida, aplicados em sistemas reais. Adicionalmente, devido à introdução massiva de tecnologia em todas as diretrizes do sistema elétrico (Bernardon et al., 2008) e com a introdução dos conceitos das Redes Elétricas Inteligentes, a utilização de meios digitais torna-se ainda mais consolidada. Com isso, verifica-se que os processos operacionais se tornam ainda mais dinâmicos, devido à agilidade proporcionada por sistemas automáticos.

A eficiência do mecanismo operacional descrito é incontestável. Entretanto, verifica-se que este também dependente da ação humana, logo, o mesmo está sujeito a falhas, que por sua vez, estão relacionadas à responsabilidade e a quantidade de informações que devem ser processadas em um curto intervalo de tempo. Dessa forma, além do ensino técnico, destaca-se a importância em despertar habilidades pessoais, apresentando ao futuro profissional, problemas relacionados ao conteúdo teórico, e propondo a busca por soluções. Porém, devido ao conservadorismo envolvido no processo de aprendizagem acadêmica, é comum que a formação de novos engenheiros seja baseada na percepção do docente em relação ao conteúdo programático, não havendo o incentivo em buscar novos conhecimentos.

\section{A APLICAÇÃO DO MÉTODO DE APRENDIZAGEM BASEADA EM PROBLEMAS (PBL)}

Muito se discute acerca de introdução de metodologias e desenvolvimento de projetos baseados em problemáticas, aos quais se destacam os trabalhos de Angelo \& Bertoni (2010). Basicamente, a metodologia PBL (do inglês Problem Based Learning) consiste na descentralização do professor no ambiente acadêmico, direcionando o foco de aprendizagem para problemas relacionados a projetos reais, estimulando assim, o aluno a se tornar mais autônomo e dinâmico.

Historicamente, a metodologia PBL foi introduzida no ensino clínico, com intuito de desenvolver o estudo individual, o convívio social e o trabalho em equipe. Porém, diante dos bons resultados obtidos, esta nova técnica de ensino passou a ser utilizada em diversas áreas, inclusive na engenharia, como apresentado por Pires (2010), Notaro (2001) e Burini (2003). 
Uma das principais vertentes deste método é despertar no discente a vontade de pesquisar conteúdos extras, considerando que o docente não é capaz de lhe ensinar "tudo" (Behrens 2015). Entretanto, é preciso que o aluno saiba discernir e considerar apenas o que é de maior relevância para resolver seus problemas e sanar parte de suas dúvidas, características essências em um processo ideal de aprendizagem. Ainda, autores como Camp (2016) e Demo (2001), destacam que nesta metodologia, ao contrário das técnicas pedagógicas tradicionais ${ }^{1}$, o conhecimento obtido pelo aluno é retido por mais tempo, devido as atividades práticas desenvolvidas ao longo do processo de pesquisa por soluções e as lembranças relacionadas ao convívio social ao decorrer dos estudos.

No contexto do presente manuscrito, a metodologia PBL foi introduzida com o objetivo de aprimorar a formação de novos profissionais, tornando-os proativos, diante das responsabilidades que irão assumir. Dessa forma, o aluno é colocado diante de algumas situações em que ele seja responsável pela pesquisa e desenvolvimento de novas soluções, ou até mesmo no aprimoramento de metodologias clássicas.

Para tanto, alguns conceitos da metodologia pedagógica PBL são utilizados, conforme fomentados pelas pesquisas de Perrenoud (1999) e Linge (2006). Neste sentido, destaca-se, por exemplo, a criação de um grupo de alunos investigadores, os quais tem a responsabilidade de pesquisar as metodologias, analisar cada viabilidade diante do problema proposto e desenvolver em laboratório, a partir da ferramenta apresentada, $\mathrm{o}$ equivalente reduzido da RD em questão. Para auxiliar nas pesquisas, criou-se uma comissão de professores, cuja principal função é relacionar as teorias de aprendizagem com o problema proposto. Ademais, o professor possui o papel apenas de incentivador, não havendo nenhuma relação de ajuda, quando direcionados a pesquisa e estudo individual de cada aluno.

\section{A FERRAMENTA EDUCACIONAL}

Considerando os fatores apresentados, desenvolveu-se uma ferramenta educacional, com intuito de direcionar aos alunos de iniciação científica, alguns problemas relacionados ao planejamento e operação de uma RD que não são abordados durante o período de graduação. Com isso, pretende-se fomentar os conceitos abordados anteriormente, como por exemplo, proporcionar à vivência de cenários e procedimentos próximos a realidade para o que aluno aprenda, a partir de um novo ponto de vista.

Para tanto, a ferramenta em questão conta com três elementos que são fundamentais no processo de ensino, pesquisa e desenvolvimento prático/teórico, são eles: rede de testes experimentais, sistema de supervisão e controle (SCADA) e um painel didático interativo. Estes, por sua vez, possuem função de representar parte dos principais elementos de controle operacional de uma $\mathrm{RD}$, em escala laboratorial. Por ser uma ferramenta construtiva, ou seja, composta por diversos elementos, destaca-se a vantagem de pesquisa, necessária para que a ferramenta seja capaz de auxiliar nos estudos considerados.

Ressalta-se também, que a introdução desta nova ferramenta de ensino não estingue a utilização de softwares, nem mesmo deixa de abordar as metodologias clássicas envolvidas neste contexto. Sendo assim, ela apenas agrega na aprendizagem no manuseio e na vivência de alguns problemas que, possivelmente, farão parte do cotidiano daqueles que desejam ingressar carreira no segmento de distribuição de energia elétrica.

A seguir, as ferramentas citadas são apresentadas brevemente, abordando suas características práticas no contexto em que estas são aplicadas, e suas principais

\footnotetext{
${ }^{1}$ Metodologia Tradicional de Ensino: Método baseado em conteúdo programado, com avaliação teórica, a qual exige temporariamente da capacidade de pesquisa e absorção de conhecimento por parte do aluno, proporcionando esquecimento em um curto espaço de tempo.
}

V. $14 \mathrm{~N}^{\circ}$ 2, dezembro, 2016 
vantagens em relação ao método clássico de ensino. Importante ressaltar que, devido ao escopo deste trabalho não se tratar do desenvolvimento da ferramenta, não serão apresentados os meios de prototipagem, nem mesmo o processo necessário para a obtenção desta, especificados de maneira detalhada em Santos (2016).

\subsection{Rede para Testes Experimentais}

A rede para testes experimentais tem por objetivo representar algumas das principais características operacionais de uma RD como, por exemplo, chaves, dispositivos de proteção e leitura.

Esta pode ser considerada uma das principais ferramentas, dentre todas as desenvolvidas. Isso porque, é a partir dela que são coletadas todas as informações necessárias e relevantes para o estudo em questão, como a tensão, corrente e consumo energético $(\mathrm{kW})$, além da realização de manobras, através da mudança de estado das chaves.

Sua prototipagem laboratorial é feita a partir de equipamentos de baixo custo, como relés de proteção digital simples, elementos de leitura e aquisição de dados. A escolha por esse tipo de equipamento deve-se, principalmente, ao fato destes proporcionarem o comando remoto, através do SCADA e do painel didático. Além disso, também torna-se possível a leitura e amostragem das grandezas elétricas da rede (tensão, corrente, potência, dentre outras), a partir da comunicação serial com um computador.

Outra função da rede de testes é a representação do carregamento energético da RD em estudo. Para essa função, optou-se pela utilização de banco de cargas resistivas, sendo este capaz de proporcionar, em escala reduzida, os mesmos efeitos ativos de uma RD em tamanho real. Assim, cabe ao aluno designar os valores referentes as resistências que serão alocadas em cada AL, de acordo com o carregamento apresentado.

\subsection{Sistema de Supervisão e Controle em Tempo Real (SCADA)}

Outra ferramenta utilizada neste trabalho é o sistema de supervisão e controle em tempo real (SCADA), um instrumento muito bem conceituado pelas redes elétricas inteligentes. Sua função, neste trabalho, é representar o diagrama unifilar da rede de testes desenvolvida em laboratório, tornando possível o controle das chaves do sistema (relés de proteção) de maneira remota, além da leitura, em tempo real, das grandezas elétricas do sistema.

\subsection{Painel Didático Interativo}

Embora o painel didático possua a mesma função que o SCADA, ele representa o diagrama unifilar da rede de uma forma diferente. Isso porque, a utilização de blocos de comando e controle tornam a implementação, em laboratório, mais palpável e atrativa, devido a possibilidade deste controlar diretamente as chaves e o carregamento do sistema. 


\subsection{Vantagens Educacionais}

Quando comparado com métodos tradicionais de ensino, como a utilização de softwares e disciplinas profissionais conteudistas ${ }^{2}$, verifica-se que a ferramenta em estudo proporciona, ao aluno, a vivência de situações que diferem do ambiente clássico de ensino. Além disso, ele é constantemente colocado a trabalhar com equipamentos e técnicas que dificilmente são abordadas durante a graduação, como por exemplo, a parametrização de relés de proteção e um SCADA. Outras vantagens como a utilização massiva de métodos matemáticos, empregados na operação de uma RD, a autonomia do aluno enquanto pesquisador, a busca por soluções e o aprimoramento de métodos, são as principais vantagens educacionais que a ferramenta proporciona, em relação a metodologia clássica de ensino.

A figura apresentada a seguir (Figura 1), ilustra as ferramentas descritas, em funcionamento, durante os testes realizados em laboratório. A partir dela, verifica-se o painel didático mais a frente (3), ao fundo estão os relés de proteção utilizados na representação de chaves e na leitura de corrente (2(a)) e, na parte superior da bancada, o banco de cargas resistivas (2(b)) e o computador operando com o SCADA em tempo real (1).

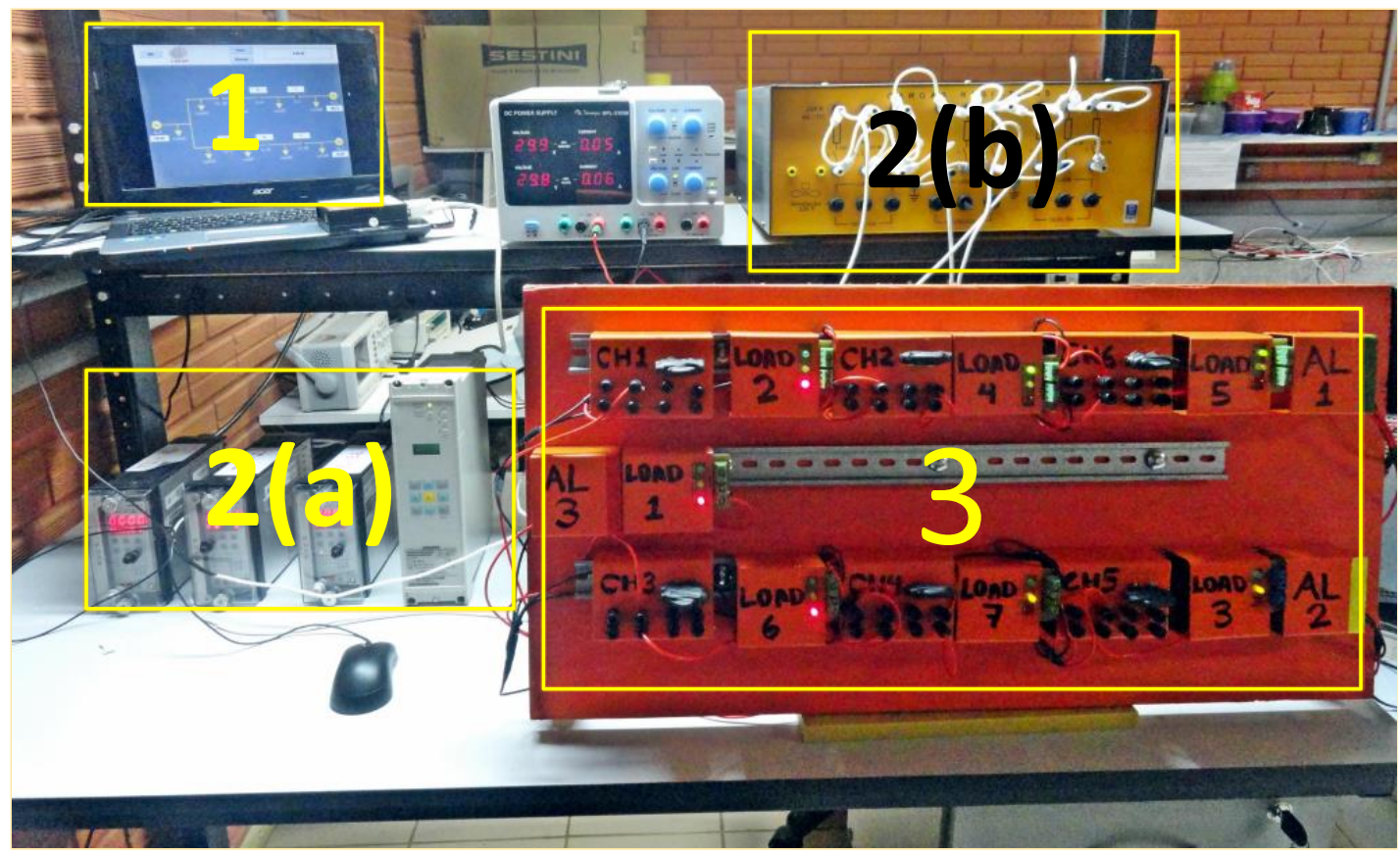

Figura 1 - Ferramenta experimental: 1 - SCADA; 2 - (a) rede de testes e (b) banco de cargas; 3 - painel didático interativo.

\section{A FERRAMENTA APLICADA AO ENSINO}

A fim de verificar a viabilidade de inserção da ferramenta apresentada no ensino operacional de sistemas de distribuição de energia elétrica, foi proposto aos alunos de iniciação científica, o desenvolvimento de um estudo voltado a otimização ${ }^{3}$ de uma RD.

\footnotetext{
${ }^{2}$ Ensino profissional conteudista - Prática de ensino que valoriza a passagem de uma vasta quantidade de informação aos alunos, sem que haja a preocupação da absorção das informações transmitidas, nem mesmo com o desenvolvimento lógico (Fernandes, 1998).

${ }^{3}$ Otimização: Otimizar o desempenho de uma rede de distribuição consiste, basicamente, em reduzir os níveis de alguns critérios que sejam prejudiciais para o sistema, como por exemplo, altos índices de perdas de energia ou baixo nível de confiabilidade.
} 
Para isso, os alunos desenvolveram o projeto, em um ambiente controlado, de um sistema idêntico ao que foi utilizado nos estudos desenvolvidos pelo professor, a partir de uma ferramenta computacional. Coube ao aluno escolher as metodologias de redução de rede, de otimização, dentre outros métodos necessários, sem ter o conhecimento dos que foram utilizados pelo docente. Além disso, este teve a função de desenvolver o SCADA e ajustar os blocos de comando do painel didático.

A forma de avaliação da ferramenta foi feita através de duas provas teóricas, sendo uma realizada ao término dos estudos, a partir de métodos clássicos de ensino, e outra após o desenvolvimento da ferramenta laboratorial. Ambas avaliações possuíam as mesmas questões, todas elas direcionadas a conceitos teóricos e práticos, relacionados a operação de sistemas de distribuição de energia elétrica. Vale ressaltar, que a avaliação teórica possui um teor de confiabilidade maior em relação à comparação dos resultados dos estudos desenvolvidos entre professor e alunos. Isso porque, apesar de ambos analisarem o mesmo sistema elétrico, os estudos tinham objetivos diferentes. Sendo assim, os alunos tinham a função de reduzir a RD, assemelhando-se ao estudo do professor, apenas a parte de otimização do sistema e aos métodos necessários para tal.

As questões abordadas abrangeram três grandes áreas, são elas: processo operacional, metodologias matemáticas, e a teoria envolvida ao tema de sistemas de distribuição. A Tabela 1 apresenta, de maneira sintetizada, a comparação entre os resultados obtidos nas avaliações realizadas. Quanto a Figura 2, esta apresenta graficamente o desempenho dos alunos em relação aos métodos de aprendizagem utilizados.

Tabela 1 - Resultado percentual das avaliações das metodologias de ensino.

\begin{tabular}{c|ccc}
\hline \multirow{2}{*}{ Método de Ensino } & \multicolumn{3}{|c}{ Temas Avaliados } \\
\cline { 2 - 4 } & Processo Operacional & Métodos Matemáticos & Teoria \\
\hline Clássico & $68,85 \%$ & $80,25 \%$ & $83,65 \%$ \\
\hline Ferramenta Educ. & $90,10 \%$ & $83,20 \%$ & $88,52 \%$ \\
\hline
\end{tabular}

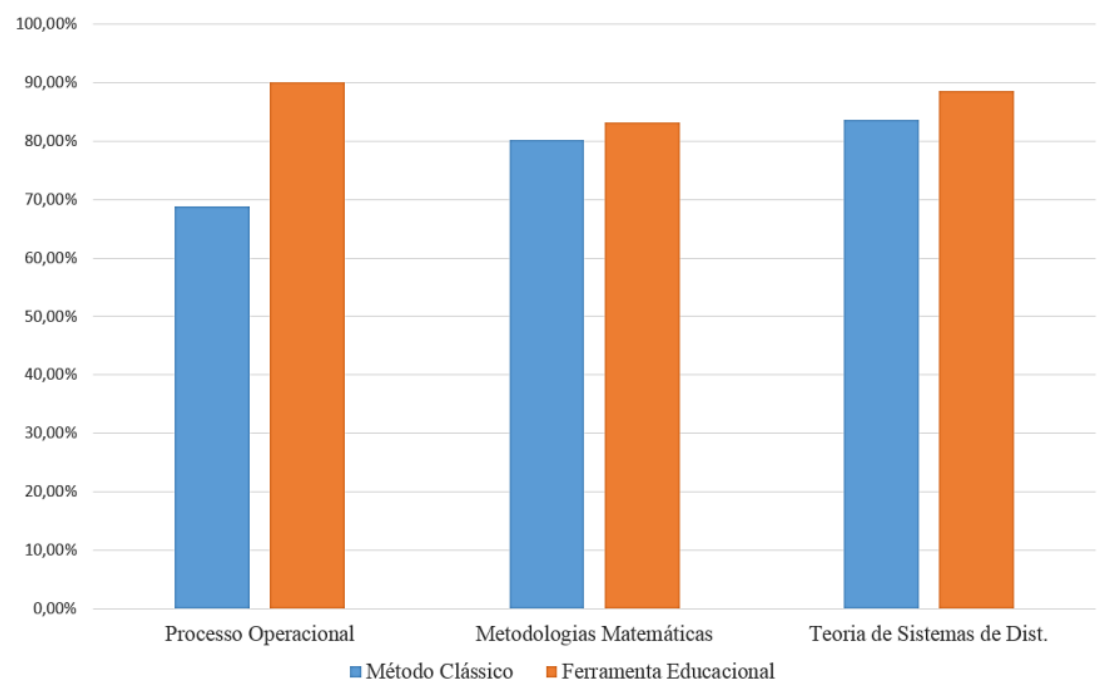

Figura 2 - Desempenho avaliativo das metodologias de ensino aplicada. 


\section{CONSIDERAÇÕES FINAIS}

A importância de equipamentos didáticos no desenvolvimento profissional é incontestável. Em decorrência disto, em um processo de ensino, a inclusão de novas ferramentas favorece a melhora no desempenho acadêmico, frente ao dinamismo proporcionado através de sua utilização. Essas ferramentas podem assumir diversas formas, sendo softwares de modelagem e simulação uma das mais comuns.

Quando direcionados ao contexto deste trabalho, verifica-se a utilização massiva deste tipo de instrumento, aliado ao processo de ensino conteudista. Isso ocorre devido a falta de equipamentos práticos, que possibilitem a reprodução de situações, metodologias e teorias em um ambiente controlado. Outro fator inerente, diz respeito ao conservadorismo, relacionado ao processo de ensino, fazendo de ferramentas computacionais, o principal instrumento de aprendizagem operacional.

Embora seja de extrema importância saber lidar com este tipo de ferramenta, o ensino técnico não deve ser engessado apenas na reprodução digital de metodologias prontas. Com isso, é fundamental inovar em processos pedagógicos e instrumentos laboratoriais, como forma de tornar o ensino mais atrativo. Em decorrência disso, o presente trabalho teve, como principal vertente, sanar parte desta problemática, através da criação e implementação de uma ferramenta educacional, aplicada ao ensino operacional de uma RD.

Para avaliar a viabilidade de inserção, no ensino, da ferramenta desenvolvida, houve a necessidade em realizar um processo avaliativo. Neste, um mesmo grupo de alunos foi testado, ao término dos dois procedimentos práticos, sendo o primeiro, a metodologia clássica, e o último, a ferramenta educacional. Dos resultados, verificou-se que a introdução deste novo método de ensino teve um impacto positivo, em todas as vertentes avaliadas. Dentre os tópicos que foram analisados, verificou-se uma mudança significativa do conhecimento dos alunos em relação ao processo operacional de RDs. Ademais, melhorias também foram obtidas, do ponto de vista pedagógico. Parte desses resultados já era esperado, uma vez que, ao comparar o ensino teórico e a aprendizagem de metodologias matemáticas, verifica-se que ambos os processos analisados são semelhantes, diferenciando apenas nos meios de busca e aprendizagem. Contudo, no processo operacional, verifica-se que a melhora considerável obtida, deve-se principalmente, ao manuseio de equipamentos e desenvolvimento de uma ferramenta totalmente interativa e didática, a qual exige conhecimento para sua prototipagem laboratorial ótima.

Com isso, conclui-se que a aplicação da ferramenta educacional desenvolvida é satisfatória, devido à demonstração da possibilidade de avaliar, de forma precisa, processos operacionais de uma RD em ambiente acadêmico.

\section{AGRADECIMENTOS}

Os autores deste trabalho agradecem ao apoio técnico e financeiro do Conselho Nacional de Desenvolvimento Científico e Tecnológico (CNPq), Fundação de Amparo à Pesquisa do Estado do Rio Grande do Sul (FAPERGS) e Coordenação de Aperfeiçoamento de Pessoal de Nível Superior (CAPES). 


\section{BIBLIOGRAFIA}

ANGELO, M. F.; BERTONI, F. C.; SANTOS, J. A. M.; LOULA, A. Análise da aplicação do método PBL no ensino de programação em ensino em engenharia da computação. In: PBL 2010 International Conference - Problem Based Learning and Active Learning Methodologies, 2010, São Paulo, PBL 2010.

BARAN, M. E.; WU, F. F. Network reconfiguration in distribution systems for loss reduction and load balancing. IEEE Transactions on Power Delivery, v.4, n.2, p.14011407, 1989.

BERNARDON, D. P. Novos métodos para reconfiguração das redes de distribuição a partir de algoritmos de tomadas de decisão multicriteriais. Tese (Doutorado em Engenharia Elétrica) Universidade Federal de Santa Maria, 2007.

BERNARDON, D.P.; et al. Studies of Parallelism in Distribution Networks Served by Different-Source Substations. Electric Power Systems Research, v.78, n.3, p. 450-457, 2008.

BEHRENS, M. A.; MORAN, J. M.; MASETTO, M. T. Novas Tecnologias e Medicação Pedagógica. Campinas: Papirus, 2000.

BEHRENS, M. A.; Metodologia de Projetos: aprender e ensinar para a produção do conhecimento numa visão complexa. Programa Agrinho - Metodologia para a Produção do Conhecimento: da Concepção à Prática. Curitiba: SENAR - PR, 2015.

BELHOT, R. V. Reflexões e propostas sobre o "Ensinar Engenharia" para o século XXI. Tese (Livre Docência), EESC, USP, São Carlos, 1997.

BURINI, E. R. V., PINHEIRO, A. C. F. O aprendizado baseado em problemas como metodologia de ensino nos cursos de engenharia. In: Congresso Brasileiro de Ensino em Engenharia, Rio de Janeiro, 2003.

CAMP, G. Problem Based Learning: A Paradigm Shift of a Passing Fad? Medical Education Online. Disponível em: <http://www.med-ed-online.org/f0000003.htm> Acesso em: 09/08/2016.

CHANG, W. F., WU, Y. C. Optimal reliability design in an electrical distribution system via a polynomial-time algorithm. Electric Power and Energy Systems, n.25, p.659-666, 2003.

CHERKAOUI, R; BART, A.; GERMOND, A.J. Optimal configuration of electrical distribution networks using heuristic methods. Proceedings of the 11th Power Systems Computation Conference, Zurich, v.1, p.147-154, 1993.

CIVANLAR, S.; GRAINGER, J. J.; YIN, H.; LEE, S. S. H. Distribution feeder reconfiguration for loss reduction. IEEE Transactions on Power Delivery, v.3, n.3, p.1217-1223, jul. 1988.

DAS, D. Reconfiguration of Distribution System using Fuzzy Multi-Objective Approach. Electrical Power and Energy Systems, Elsevier, v.28, p.331-338, 2006.

DEMO, P. Educar pela pesquisa. Campinas, São Paulo. Autores Associados, 1997. 
DEMO, P. Pesquisa de Informação Qualitativa. Campinas Papirus, 2001.

GOEL, L.; OU, Y. Reliability worth assessment in radial distribution systems using the Monte Carlo simulation technique. Electric Power Systems Research, n.51, p;43-53, 1999.

HONG, Y. e HO, S. Determination of Network Configuration Considering Multiobjective in Distribution Systems using Genetic Algotithms. IEEE Transactions on Power Systems, v. 20, n. 2, p. 1062-1069, 2005.

KAGAN, N.; OLIVEIRA, C. C. B. Reconfiguração de sistemas de distribuição de energia elétrica através de ferramenta para solução de problemas de decisão com múltiplos objetivos e incertezas. CBA - Congresso Brasileiro de Automática, São Paulo, 1996.

LINGE, N.; PARSONS, D. Problem-Based Learning as a Effective Tool for Teaching Computer Network Desing. IEEE Transaction on Education, v.49, n.1, 2006.

MACEDO, R. J.; DUARTE, M. A.; TEIXEIRA, N. G. Novas metodologias de ensino e aprendizagem aplicadas ao curso de engenharia elétrica: o foco do ensino do século XXI, XL Congresso Brasileiro de Educação em Engenharia, Belém, 2012.

NOTARO, I. A. et al. O aprendizado baseado em problemas no curso de graduação em engenharia agrícola da UFPB. In: XXIX Congresso Brasileiro de Ensino em Engenharia, Porto Alegre, 2001.

PERRENOUD, P. Avaliação. Trad. RAMOS, Patrícia Chittoni. Porto Alegre: Artmed, 1999.

PFITSCHER, L. Reconfiguração automática das redes de energia elétrica com monitoramento em tempo real. Tese (Doutorado em Engenharia Elétrica) Universidade Federal de Santa Maria, 2013.

PIRES, M. F.; SANTOS, J. A. M. Utilização do método PBL no ensino de sistemas operacionais e redes de computadores. In: PBL 2010 International Conference Problem Based Learning and Active Learning Methodologies, 2010, São Paulo, PBL 2010.

SANTOS, C.; BOASKI, F. B.; MELlO, A. P. C.; BERNARDON, D. P. Desenvolvimento de Rede Experimental para Análise de Métodos de Reconfiguração Automática de Redes de Distribuição de Energia Elétrica. SBSE - Simpósio Brasileiro de Sistemas Elétricos, Natal, Rio Grande do Norte, 2016.

SU, C.T.; LEE, C.S. Network reconfiguration of Distribution Systems using Improved Mixed-Integer Hybrid Differential Evolution. IEEE Transactions on Power Delivery, v.18, No.3, p.1022-1027, July 2003.

TSAO, T.; CHANG, H. C. Composite reliability evaluation model for different types of bdistribution systems. IEEE Transactions on Power Systems, v.18, n.2, p.924-930, 2003. 